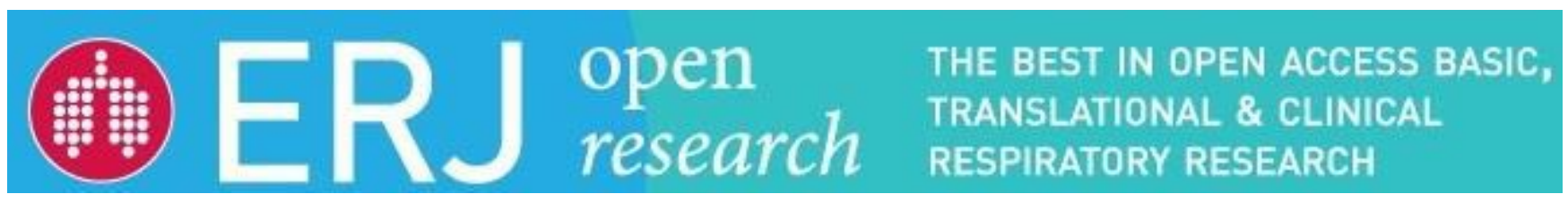

\title{
Early View
}

Original research article

\section{Predicting In-hospital Death in Pneumonic COPD exacerbation via BAP-65, CURB-65, and Machine Learning}

Akihiro Shiroshita, Yuya Kimura, Hiroshi Shiba, Chigusa Shirakawa, Kenya Sato, Shinya Matsushita, Keisuke Tomii, Yuki Kataoka

Please cite this article as: Shiroshita A, Kimura Y, Shiba H, et al. Predicting In-hospital Death in Pneumonic COPD exacerbation via BAP-65, CURB-65, and Machine Learning. ERJ Open Res 2021; in press (https://doi.org/10.1183/23120541.00452-2021).

This manuscript has recently been accepted for publication in the ERJ Open Research. It is published here in its accepted form prior to copyediting and typesetting by our production team. After these production processes are complete and the authors have approved the resulting proofs, the article will move to the latest issue of the ERJOR online.

Copyright $($ The authors 2021. This version is distributed under the terms of the Creative Commons Attribution Non-Commercial Licence 4.0. For commercial reproduction rights and permissions contact permissions@ersnet.org 
Predicting In-hospital Death in Pneumonic COPD exacerbation via BAP-65, CURB65, and Machine Learning

Akihiro Shiroshita ${ }^{1}$, MD, MPH; Yuya Kimura ${ }^{2}$, MD; Hiroshi Shiba ${ }^{3}$, MD; Chigusa Shirakawa ${ }^{4}$, MD; Kenya Sato ${ }^{5}$, MD; Shinya Matsushita ${ }^{5}$, MD; Keisuke Tomii ${ }^{4}$, MD, PhD; Yuki Kataoka ${ }^{6,7,8}, \mathrm{MD}, \mathrm{MPH}, \mathrm{DrPH}$

${ }^{1}$ Department of Respiratory Medicine, Ichinomiyanishi Hospital, Japan

${ }^{2}$ Clinical Research Center, Department of Respiratory Medicine, National Hospital Organization Tokyo National Hospital, Japan

${ }^{3}$ Post Graduate Education Center, Kameda Medical Center, Japan

${ }^{4}$ Department of Respiratory Medicine, Kobe City Medical Center General Hospital, Japan

${ }^{5}$ Department of Thoracic Medicine, Saiseikai Yokohamashi Tobu Hospital, Japan

${ }^{6}$ Department of Internal Medicine, Kyoto Min-Iren Asukai Hospital, Japan

${ }^{7}$ Section of Clinical Epidemiology, Department of Community Medicine, Kyoto University Graduate School of Medicine, Japan

${ }^{8}$ Department of Healthcare Epidemiology, Graduate School of Medicine / Public Health, Kyoto University, Japan

\section{Corresponding author:}

Akihiro Shiroshita

Department of Respiratory Medicine, Ichinomiyanishi Hospital

1 Kaimeihira, Ichinomiya, Aichi 494-0001, Japan 
E-mail: akihirokun8@gmail.com

Telephone number: $+81-80-3807-4960$

Fax number: +586-48-0077

ORCID: https://orcid.org/0000-0003-0262-459X

\section{Summary}

BAP-65, CURB-65, and the XGBoost model showed low predictive performance for inhospital death in pneumonic COPD exacerbation. Further large-scale studies with more variables are warranted to develop an ideal prognostic model. 


\begin{abstract}
Introduction

There is no established clinical prediction model for in-hospital death among patients with pneumonic chronic obstructive pulmonary disease (COPD) exacerbation. We aimed to externally validate BAP-65 and CURB-65 and to develop a new model based on the eXtreme Gradient Boosting (XGBoost) algorithm.
\end{abstract}

\title{
Methods
}

This multicentre cohort study included patients aged $\geq 40$ years with pneumonic COPD exacerbation. The input data were age, sex, activities of daily living, mental status, systolic and diastolic blood pressure, respiratory rate, heart rate, peripheral blood eosinophil count, and blood urea nitrogen. The primary outcome was in-hospital death. BAP-65 and CURB-65 underwent external validation using the area under the receiver operating characteristic curve (AUROC) in the whole dataset. We used XGBoost to develop a new prediction model. We compared the AUROCs of XGBoost with that of BAP-65 and CURB-65 in the test dataset using bootstrap sampling.

\section{Results}

We included 1190 patients with pneumonic COPD exacerbation. The in-hospital mortality was $7 \%$ (88/1190). In the external validation of BAP-65 and CURB-65, the AUROCs (95\% confidence interval [CI]) of BAP-65 and CURB-65 were 0.69 (0.660.72, and 0.69 (0.66-0.72), respectively. XGBoost showed an AUROC of $0.71(0.62-$ 0.81) in the test dataset. There was no significant difference in the AUROCs of 
XGBoost vs BAP-65 (absolute difference, 0.054; 95\% CI, -0.057-0.16) or vs CURB-65 (absolute difference, 0.0021; 95\% CI, -0.091-0.088).

\section{Conclusion}

BAP-65, CURB-65, and XGBoost showed low predictive performance for in-hospital death in pneumonic COPD exacerbation. Further large-scale studies including more variables are warranted.

Keywords: Chronic obstructive pulmonary disease, Machine Learning, Pneumonia, Prognosis 


\section{INTRODUCTION}

Chronic obstructive pulmonary disease (COPD) is a common respiratory disease that is characterised by airflow limitation due to chronic inflammation of the airways and lungs [1]. Patients with COPD often experience acute worsening of baseline symptoms, and with coexisting consolidation (pneumonic COPD exacerbation) on chest imaging, mortality is increased compared to non-pneumonic COPD exacerbation [2]. A previous study suggested that pneumonic COPD exacerbation might have a different inflammation profile from non-pneumonic COPD exacerbation [3,4].

CURB-65 (confusion, blood urea nitrogen $>19 \mathrm{mg} / \mathrm{dL}$, respiratory rate $\geq 30$ breaths/min, systolic blood pressure $<90 \mathrm{mmHg}$ or diastolic blood pressure $\leq 60$ $\mathrm{mmHg}$, and age $\geq 65$ years) is a simple prediction model in patients with communityacquired pneumonia and has been validated internally and externally [5,6]. On the other hand, BAP-65 (blood urea nitrogen $\geq 25 \mathrm{mg} / \mathrm{dL}$, altered mental status, heart rate $\geq$ 109 beats/min, and age $\geq 65$ years) is an easily computable prediction model in patients with COPD exacerbation, that has shown good performance in internal validation and external validation cohorts $[7,8]$. However, we could not evaluate how many patients with pneumonic COPD exacerbation were included in those studies. Another study showed that CURB-65 had poor predictive ability for death in pneumonic COPD exacerbation [9]. To date, there is no established clinical prediction model specifically for the population with pneumonic COPD exacerbation. It is also unclear whether BAP-65 and CURB-65 can be applied to patients with pneumonic COPD exacerbation [10]. Our study had two purposes: (i) the external validation of BAP-65 and CURB-65 for predicting in-hospital death among patients with pneumonic COPD exacerbation, and (ii) the development of a 
high-performance clinical prediction model using a modern machine learning algorithm that is gaining ground in the medical field [11].

\section{METHODS}

\section{Study design}

Our study was a multicentre retrospective cohort study conducted across five acute care hospitals in Japan. To maximise patient capture, patient data were collected during different periods in each hospital between April 1, 2008, and July 31, 2020.

Pneumonic COPD exacerbation is diagnosed when the criteria for both pneumonia and COPD exacerbation are met $[12,13,14]$. To select patients with pneumonic COPD exacerbation, we used the validated algorithm based on the 10th revision of the International Classification of Diseases and Related Health Problems (eFigure 1) [14]. First, patients aged $\geq 40$ years who had both pneumonia and COPD exacerbation were selected. Patients with other differential diagnoses mimicking pneumonic COPD exacerbation were excluded, including heart failure, pneumothorax, asthma exacerbation, and obstructive pneumonia.

This study was approved by the institutional review board of each hospital (approval number, 200811). This article was reported according to the Transparent Reporting of a multivariable prediction model for Individual Prognosis Or Diagnosis (TRIPOD) statement (eTable1) [15].

\section{Input and output data}

The following input data on the day of admission were collected from the data warehouse or electrical medical records in each hospital: age, sex, the activities of daily living status (full support or not), mental status (altered mental status or not), vital signs (systolic and 
diastolic blood pressure, respiratory rate, and heart rate), laboratory results (peripheral blood eosinophil count and blood urea nitrogen), and presence of tracheal intubation. Activities of daily living were defined as full support when the Barthel index was zero, and altered mental status was defined as a Japan Coma Scale score $\geq 1$. These two variables are used for administrative purposes in the Japanese original case-mix classification system or Diagnosis Procedure Combination [16,17]. The Ministry of Health, Labour, and Welfare regularly evaluates the trend, quality, and cost of the health care system using Diagnosis Procedure Combination data. We extracted data from the database containing Diagnosis Procedure Combination data submitting the anonymised patient data to the Ministry of Health, Labour, and Welfare. Our variable selection was based on existing clinical prediction models of pneumonia or COPD exacerbation $[5,7,18]$. We did not collect data on other comorbidities from the Diagnosis Procedure Combination database because these variable codes were not fully externally validated. The primary outcome was in-hospital death which was derived from the electrical medical records in each hospital.

\section{Statistical analysis}

The study process is illustrated in Figure 1. Patient characteristics were summarised as means for continuous variables and as percentages for categorical variables. All statistical analyses were performed using R software version 4.0.2 (R Foundation for Statistical Computing, Vienna, Austria). The scripts are available in the GitHub repository (https://github.com/AkihiroShiroshita/Prediction-model-for-PnuemonicCOPD-exacerbation.git). 


\section{External validation of BAP-65 and CURB-65}

We conducted the external validation of BAP-65 and CURB-65 with respect to the entire dataset to evaluate their performance in a large sample size. We calculated the sensitivity and specificity using each total score as the cut-off point. To assess the calibration ability, we summarised the mortality according to each total risk score. To assess the discriminatory ability of the two models, we calculated the area under the receiver operating characteristic curve (AUROC). We used multiple imputation to cope with missing data [19]. We created a total of 100 datasets using multiple imputation with chained equations and calculated the AUROC within each dataset. Thereafter, we combined the estimates of AUROC using Rubin's combining rule [20,21].

\section{Model development via machine learning}

We used the eXtreme Gradient Boosting (XGBoost) algorithm to develop a clinical prediction model for in-hospital death among patients with pneumonic COPD exacerbation. The XGBoost algorithm is a powerful ensemble method of machine learning that combines a set of weak learners of the decision tree [22]. Its parallel computation enables the efficient and accurate development of a prediction model. Because it extracts variable importance, imputation of missing data, scaling, or normalisation is not required. What is required in the algorithm is the proper tuning of the hyperparameters, which are parameters that control the behaviour of the model. In our study, the original data were first partitioned into training and test datasets. We used the stratified sampling method with a 7:3 ratio for data splitting, which allowed the two datasets to have similar in-hospital mortality. Second, we developed prediction models using a training dataset. We performed a grid search with 4-fold cross-validation to obtain the optimal hyperparameters for maximising the mean AUROC (eFigure2) [22]. In the 
grid search, the hyperparameter candidates for max_depth (maximum tree depth) was $\{2$, $4,6,8,10\}$, and min_child_weight (minimum degree of impurity needed in a node) was $\{1,2,3,4,5\}$. After fixing max_depth and min_child_weight, we searched the maximum number of trees based on the cross-validation. We set the remaining hyperparameters as default. Third, for external validation, we validated the trained model using the test dataset. We used the AUROC as an index to validate the model. Finally, the importance of the variables based on the impurity metric was plotted. Impurity is the degree of misclassification. It displays the degree to which each input data influences the output in our XGBoost model.

\section{Model comparison}

We compared the model performances of the three prediction models using the test dataset to allow comparison on a one-to-one basis. To evaluate the discriminatory performance, we described the ROC curves of the three prediction models. Thereafter, we estimated the differences in AUROCs using bootstrap sampling (BAP-65 vs XGBoost, and CURB-65 vs XGBoost) [23]. The XGBoost model can take into account missing data while BAP-65 and CURB-65 cannot. The XGBoost model used the whole test dataset while BAP-65 and CURB-65 only used the patient data without missing values. 


\section{RESULTS}

The patient selection flowchart is shown in Figure 1. We initially selected 1431 patients. After excluding 241 patients with other diagnoses, 1190 patients with pneumonic COPD exacerbation were included in our analysis. Patient characteristics are summarised in Table 1 . The in-hospital mortality rate was $88 / 1190$ (7\%). The Number of intratracheal intubations was 16/1190 (1\%), and median length of hospital stay was 12 (interquartile range: $8-18)$ days.

\section{External validation of BAP-65 and CURB-65}

Table 2 presents the summary of the number of patients with each total score. The calibration performances of both prediction models were low. The AUROC of BAP-65 was 0.69 (95\% confidence interval [CI]: 0.66 to 0.72$)$ and that of CURB-65 was 0.69 (95\% CI: 0.66 to 0.72 ). The discriminatory performance of both prediction models was also low.

\section{Model development via machine learning}

Based on the results of the grid search, we set up the hyperparameters as follows: max_depth $($ maximum tree depth $)=4$, min_child_weight (minimum degree of impurity needed in a node $)=2$, eta (learning rate $)=0.1$, subsample (the proportion of cases to be randomly sampled for each tree) $=0.8$, colsample_bytree (the proportion of predictor variables sampled for each tree $)=0.8$, gamma (minimal loss to expand on a leaf node $)=$ 0, lambda (L2 regularisation term on weights) $=1$, alpha (L1 regularisation term on weights $)=0$, scale_pos_weight (balance of positive and negative weights) $=1$, and maximum number of trees $=37$. Cross-validation of the developed model revealed a mean AUROC of 0.76, and external validation in the test dataset revealed an AUROC of 0.72 (95\% CI: 0.62 to 0.82). Feature importance is illustrated in Figure 2, and it revealed that 
blood urea nitrogen was the most important factor for predicting in-hospital death. Systolic blood pressure and altered mental status also had important roles in the XGBoost model. On the contrary, activities of daily living and sex showed little importance.

\section{Model comparison}

We performed model comparisons using the test data. The XGBoost model used the whole test dataset $(\mathrm{N}=357)$, while BAP-65 and CURB-65 used the data of 314 and 281 patients, respectively, because of missing values. Figure 3 shows the ROC curves of the BAP-65, CURB-65, and XGBoost models. There was no significant difference in AUROCs between the XGBoost model and BAP-65 (absolute difference, 0.054; 95\% CI: -0.057 to 0.16) or between the XGBoost model and CURB-65 (absolute difference, 0.0021; 95\% CI: -0.091 to 0.088$)$.

\section{DISCUSSION}

Our study revealed that contrary to the study results for either pneumonia or COPD exacerbation, all three models (BAP-65, CURB-65, and XGBoost model) had low discriminatory ability for predicting in-hospital death among patients with pneumonic COPD exacerbation. Further large-scale studies are needed to develop a specific clinical prediction model for pneumonic COPD exacerbation.

The two simple scoring systems, i.e. BAP-65 and CURB-65, showed low predictive performance in our dataset of patients with pneumonic COPD exacerbation. Although our study did not contrast their predictive abilities in either pneumonia or COPD exacerbation with pneumonic COPD exacerbation, CURB-65 was externally validated for the Japanese population and BAP-65 was validated for the Chinese population [24,25]. 
Contrary to the results in either pneumonia or COPD exacerbation, CURB-65 or BAP-65 was not externally validated in our patient cohort. Our results were consistent with those of a previous retrospective cohort study that revealed CURB-65 had poor performance for predicting death in pneumonic COPD exacerbation while it had high performance for non-pneumonic COPD exacerbation [26]. Our target population included patients with a specific category of pneumonic COPD exacerbation. The disease spectrum of pneumonic COPD exacerbation which fulfils the diagnostic criteria for both pneumonia and COPD exacerbation, may differ from that of COPD exacerbation and pneumonia. A specific clinical prediction model for pneumonic COPD exacerbation is warranted.

A strength of our study was the use of a powerful machine learning technique that can overcome the drawbacks of the development processes of BAP-65 and CURB65. However, the XGBoost model also had a low predictive performance for in-hospital deaths in pneumonic COPD exacerbation. Contrary to the recursive partition that was used in the development of BAP-65, the XGBoost model avoids model instability [5,27]. In addition, unlike logistic regression, which was used in the development of CURB-65, the XGBoost model is not based on the assumption of linearity and does not require the categorisation of continuous variables [5]. It can also find the optimal interaction terms between variables. Despite its high ability, our XGBoost model did not show high performance.

However, our study had several weaknesses. First, our sample may have been too small to develop internally and externally validated prediction models. The number of events required for model development is at least 10 events per variable [28]. Our input data included 10 variables, and at least 100 events were required; however, there were only about 60 events in our training dataset. Although we used the XGBoost model, which 
may require a smaller sample size, we could not overcome the problem in our dataset [29]. Second, other missing variables should have been included in the model. For example, in a previous study, the DECAF score, a simple and validated scoring system for predicting outcomes in COPD exacerbation. tended to have a higher predictive performance than CURB-65 in pneumonic COPD exacerbation [26]. However, we could not collect the values for the Extended Medical Research Council Dyspnea Scale, arterial blood gas analysis results, or atrial fibrillation because they were not routinely collected in our clinical site. They may be additional candidates for future prediction models. According to a systematic review of prediction models for COPD exacerbation, patients' baseline characteristics such as body mass index, forced expiratory volume in 1 second, and previous COPD exacerbation were used in studies with a low risk of bias [30]. These could also be additional candidates.

The feature importance plot in our study highlighted the importance of blood urea nitrogen, systolic blood pressure, and altered mental status. These variables should be included in a new clinical prediction model for pneumonic COPD exacerbation. On the contrary, activities of daily living and sex were of little importance in the model. In our study, the activities of daily living were categorised as full support or not, and this might have led to the loss of notable information. Approximately $90 \%$ of the included patients were men, which may have led to the unimportance of sex as a variable. Our study revealed some candidates for the included variables in developing a new model.

In our study, we could not conclude which clinical model was superior. In our test dataset, the number of patients was approximately 300, and the in-hospital mortality was 25 , which was much smaller than the necessary sample size for precise external validation $[31,32]$. The results of the external validation of BAP-65 and CURB-65 in the 
whole dataset showed that the predictive ability for in-hospital death could be similar between CURB-65 and BAP-65. Physicians who use either BAP-65 or CURB-65 will not have to change their practice based on our study.

Our study had several limitations. First, as we pointed out before, our sample size was small. Because we could not include additional patients after the patient enrolment period or incorporate another patient cohort, we could not address the problem. Second, only Japanese patients were included, suggesting a lack of generalisability. Third, the primary outcome in our study was in-hospital mortality, and long-term outcomes could not be evaluated. Fourth, we could not set aside an additional dataset for external validation before splitting the dataset. Because we performed external validation on the split dataset, the AUROC in the test dataset may have been overestimated. Fifth, altered mental status was evaluated based on the Japan Coma Scale. Although this scale has been widely used in Japan because of its simplicity, its accuracy has not been validated in patients with COPD. Sixth, we could not collect data on the patients' code status (Donot-intubate or not). In our study, the rate of tracheal intubation was lower than mortality. This may have decreased the generalisability of our study results to intensive care units. To overcome these limitations, large-scale studies from different regions are needed.

\section{CONCLUSION}

BAP-65, CURB-65, and the XGBoost model showed poor performance in predicting inhospital death among patients with pneumonic COPD exacerbation. Further large-scale studies with more variables are needed to develop a new prognostic model for pneumonic COPD exacerbation. 
Acknowledgement: None

Data availability statement: The datasets generated and/or analysed during the current study are not publicly available due to the privacy issues but are available from the corresponding author on reasonable request.

Conflict of interest: The authors declare no conflicts of interest.

Funding sources: Funding for the English language editing was obtained from Ichinomiyanishi Hospital. The funder played no role in the study design, study execution, data analyses, data interpretation, or decision to submit the report.

Author contributions: AS, Yuya K, HS, CS, KS, SM, KT, and Yuki K contributed to the conception and design of the work. AS, HS, CS, KS, SM, and KT contributed to data acquisition. AS, Yuya K, and Yuki K contributed to the data analysis and interpretation. AS, Yuya K, and Yuki K drafted the manuscript. All authors revised the manuscript critically and approved the final version of the manuscript. AS, Yuya K, HS, CS, KS, SM, KT, and Yuki K agreed to be accountable for all aspects of any part of the work. 


\section{References}

1. 2020 Gold Reports. Global Initiative for Chronic Obstructive Lung Disease - GOLD. https://goldcopd.org/gold-reports. Date last accessed: March 52021.

2. Saleh A, López-Campos JL, Hartl S, et al. The effect of incidental consolidation on management and outcomes in COPD exacerbations: Data from the European COPD Audit. PLoS One 2015; 10: e0134004.

3. Huerta A, Crisafulli E, Menéndez R, et al. Pneumonic and nonpneumonic exacerbations of COPD: inflammatory response and clinical characteristics. Chest. 2013; 144: 1134-1142.

4. Shiroshita A, Shiba H, Tanaka Y, et al. Effectiveness of steroid therapy on pneumonic chronic obstructive pulmonary disease exacerbation: a multicenter, retrospective cohort study. Int $\mathbf{J}$ Chron Obstruct Pulmon Dis 2020; 15: 2539-2547.

5. Lim WS, van der Eerden MM, Laing R, et al. Defining community acquired pneumonia severity on presentation to hospital: an international derivation and validation study. Thorax 2003; 58 : 377-382.

6. Ilg A, Moskowitz A, Konanki V, et al. Performance of the CURB-65 Score in predicting critical care interventions in patients admitted with community-acquired pneumonia. Ann Emerg Med 2019; 74: 60-68.

7. Tabak YP, Sun X, Johannes RS, et al. Mortality and need for mechanical ventilation in acute exacerbations of chronic obstructive pulmonary disease: development and validation of a simple risk score. Arch Intern Med 2009; 169: 1595-1602.

8. Shorr AF, Sun X, Johannes RS, et al. Validation of a novel risk score for severity of illness in acute exacerbations of COPD. Chest 2011; 140: 1177-1183.

9. Echevarria C, Steer J, Heslop-Marshall K, et al. Validation of the DECAF score to predict hospital mortality in acute exacerbations of COPD. Thorax 2016; 71: 133-140.

10. Trethewey SP, Hurst JR, Turner AM. Pneumonia in exacerbations of COPD: what is the clinical significance? ERJ Open Res 2020; 6: 00282-2019.

11. Rajkomar A, Dean J, Kohane I. Machine learning in medicine. N Engl J Med 2019; 380: 13471358.

12. Anthonisen NR, Manfreda J, Warren CP, Hershfield ES, Harding GK, Nelson NA. Antibiotic therapy in exacerbations of chronic obstructive pulmonary disease. Ann Intern Med. 1987; 106: 196-204.

13. Shindo Y, Ito R, Kobayashi D, et al. Risk factors for drug-resistant pathogens in communityacquired and healthcare-associated pneumonia. Am J Respir Crit Care Med. 2013; 188: 985995.

14. Shiroshita A, Shiba H, Tanaka Y, et al. Effectiveness of steroid therapy on pneumonic chronic obstructive pulmonary disease exacerbation: a multicenter, retrospective cohort study. Int $\mathbf{J}$ Chron Obstruct Pulmon Dis 2020; 15: 2539-2547. 
15. Collins GS, Reitsma JB, Altman DG, et al. Transparent Reporting of a multivariable prediction model for Individual Prognosis or Diagnosis (TRIPOD): the TRIPOD statement. Ann Intern Med 2015; 162: 55-63.

16. Shigematsu K, Nakano H, Watanabe $\mathrm{Y}$. The eye response test alone is sufficient to predict stroke outcome—reintroduction of Japan Coma Scale: a cohort study. BMJ Open 2013; 3: e002736.

17. Yasunaga H, Matsui H, Horiguchi H, et al. Clinical Epidemiology and Health Services Research using the Diagnosis Procedure Combination Database in Japan. Asian Pac J Dis Manage 2013; 7: $19-24$.

18. Steer J, Gibson J, Bourke SC. The DECAF Score: predicting hospital mortality in exacerbations of chronic obstructive pulmonary disease. Thorax 2012; 67: 970-976.

19. White IR, Royston P, Wood AM. Multiple imputation using chained equations: issues and guidance for practice. Stat Med 2011; 30: 377-399.

20. Toutenburg H. Rubin, D.B. Multiple imputation for nonresponse in surveys. Stat Pap 1990; 31: 180-180.

21. Snell KI, Ensor J, Debray TP, et al. Meta-analysis of prediction model performance across multiple studies: Which scale helps ensure between-study normality for the C-statistic and calibration measures? Stat Methods Med Res 2018; 27: 3505-3522.

22. Chen T, Guestrin C. XGBoost: A scalable tree boosting system 2016; arXiv:1603.02754

23. Carpenter J, Bithell J. Bootstrap confidence intervals: when, which, what? A practical guide for medical statisticians. Stat Med 2000; 19: 1141-1164

24. Usui $\mathrm{K}$, Tanaka $\mathrm{Y}$, Noda $\mathrm{H}$, et al. Comparison of three prediction rules for prognosis in community acquired pneumonia: Pneumonia Severity Index (PSI), CURB-65, and A-DROP. Nihon Kokyuki Gakkai Zasshi 2009; 47: 781-785.

25. Huang W, Cui M, Jiang Y, et al. A prospective validation of NEWS, CREWS and BAP-65 among patients with AECOPD. Chinese Journal of Nursing 2017; 12: 381-384.

26. Echevarria C, Steer J, Heslop-Marshall K, et al. Validation of the DECAF score to predict hospital mortality in acute exacerbations of COPD. Thorax 2016; 71: 133-140.

27. Li R-H, Belford GG. Instability of decision tree classification algorithms. In: Proceedings of the eighth ACM SIGKDD International Conference on Knowledge Discovery and Data Mining. New York, NY, USA: Association for Computing Machinery; 2002. p. 570-575. (KDD '02). https://doi.org/10.1145/775047.775131. Date last accessed: June 162021.

28. Riley RD, Snell KI, Ensor J, et al. Minimum sample size for developing a multivariable prediction model: PART II - binary and time-to-event outcomes. Stat Med 2019; 38: 1276-1296.

29. Floares AG, Ferisgan M, Onita D, et al. The smallest sample size for the desired diagnosis accuracy. International Journal of Oncology and Cancer Therapy 2017; 2, 13-19.

30. Bellou V, Belbasis L, Konstantinidis AK, et al. Prognostic models for outcome prediction in patients with chronic obstructive pulmonary disease: systematic review and critical appraisal. BMJ 2019; 367: 15358. 
31. Vergouwe Y, Steyerberg EW, Eijkemans MJC, et al. Substantial effective sample sizes were required for external validation studies of predictive logistic regression models. J Clin Epidemiol 2005; 58: 475-483.

32. Steyerberg EW. Validation in prediction research: the waste by data splitting. J Clin Epidemiol 2018; 103:131-133. 
Table 1. Patient characteristics

\begin{tabular}{|c|c|c|c|c|}
\hline Characteristics & $\begin{array}{l}\text { Survivors } \\
(\mathrm{N}=1102)\end{array}$ & $\begin{array}{l}\text { Non-survivors } \\
(\mathrm{N}=88)\end{array}$ & $\begin{array}{l}\text { Total } \\
(\mathrm{N}=1190)\end{array}$ & P-values \\
\hline Age (years) & $77 \pm 8$ & $80 \pm 7$ & $77 \pm 8$ & 0.006 \\
\hline Male & $974(88)$ & $85(97)$ & $1059(89)$ & 0.029 \\
\hline Full support in activities of daily living & $188(17)$ & $23(26)$ & $211(18)$ & 0.045 \\
\hline Altered mental status & $132(12)$ & $39(44)$ & $171(14)$ & $<0.001$ \\
\hline Missing data & $8(1)$ & $0(0)$ & $8(1)$ & \\
\hline Systolic blood pressure $(\mathrm{mmHg})$ & $133 \pm 26$ & $125 \pm 25$ & $132 \pm 26$ & 0.010 \\
\hline Missing data & $149(14)$ & $5(6)$ & $154(13)$ & \\
\hline Diastolic blood pressure $(\mathrm{mmHg})$ & $75 \pm 17$ & $72 \pm 16$ & $75 \pm 17$ & 0.130 \\
\hline Missing data & $155(14)$ & $5(6)$ & $160(13)$ & \\
\hline Respiratory rate (breaths/minutes) & $25 \pm 6$ & $27 \pm 7$ & $25 \pm 7$ & $<0.001$ \\
\hline Missing data & $219(20)$ & $8(9)$ & $227(19)$ & \\
\hline Heart rate (beats/minutes) & $102 \pm 19$ & $106 \pm 21$ & $102 \pm 19$ & 0.061 \\
\hline
\end{tabular}




\begin{tabular}{|c|c|c|c|c|}
\hline Missing data & $142(13)$ & $3(3)$ & $145(12)$ & \\
\hline Blood urea nitrogen $(\mathrm{mg} / \mathrm{dL})$ & $20 \pm 11$ & $30 \pm 21$ & $21 \pm 12$ & $<0.001$ \\
\hline Missing data & $13(1)$ & $1(1)$ & $14(1)$ & \\
\hline Blood eosinophil count (counts $/ \mu \mathrm{L}$ ) & $99 \pm 169$ & $53 \pm 123$ & $96 \pm 167$ & 0.068 \\
\hline Missing data & $409(37)$ & $41(47)$ & $450(38)$ & \\
\hline
\end{tabular}

Data are presented as mean \pm standard deviation or as number (\%) 
Table 2. Risk scores and in-hospital mortality of BAP-65 and CURB-65

\begin{tabular}{lll}
\hline Risk scores & Patients (number, \%) & In-hospital mortality (number, \%) \\
\hline BAP-65 Class & & \\
\hline 1 & 20 & $0(0)$ \\
2 & 455 & $20(4)$ \\
3 & 404 & $27(7)$ \\
4 & 120 & $32(27)$ \\
5 & 22 & $5(23)$ \\
\hline CURB-65 & & \\
\hline 0 & 21 & $0(0)$ \\
1 & 306 & $16(5)$ \\
2 & 351 & $14(4)$ \\
3 & 191 & $31(16)$ \\
4 & 57 & $13(23)$ \\
5 & 8 & $2(25)$ \\
\hline
\end{tabular}




\section{Figure Legends}

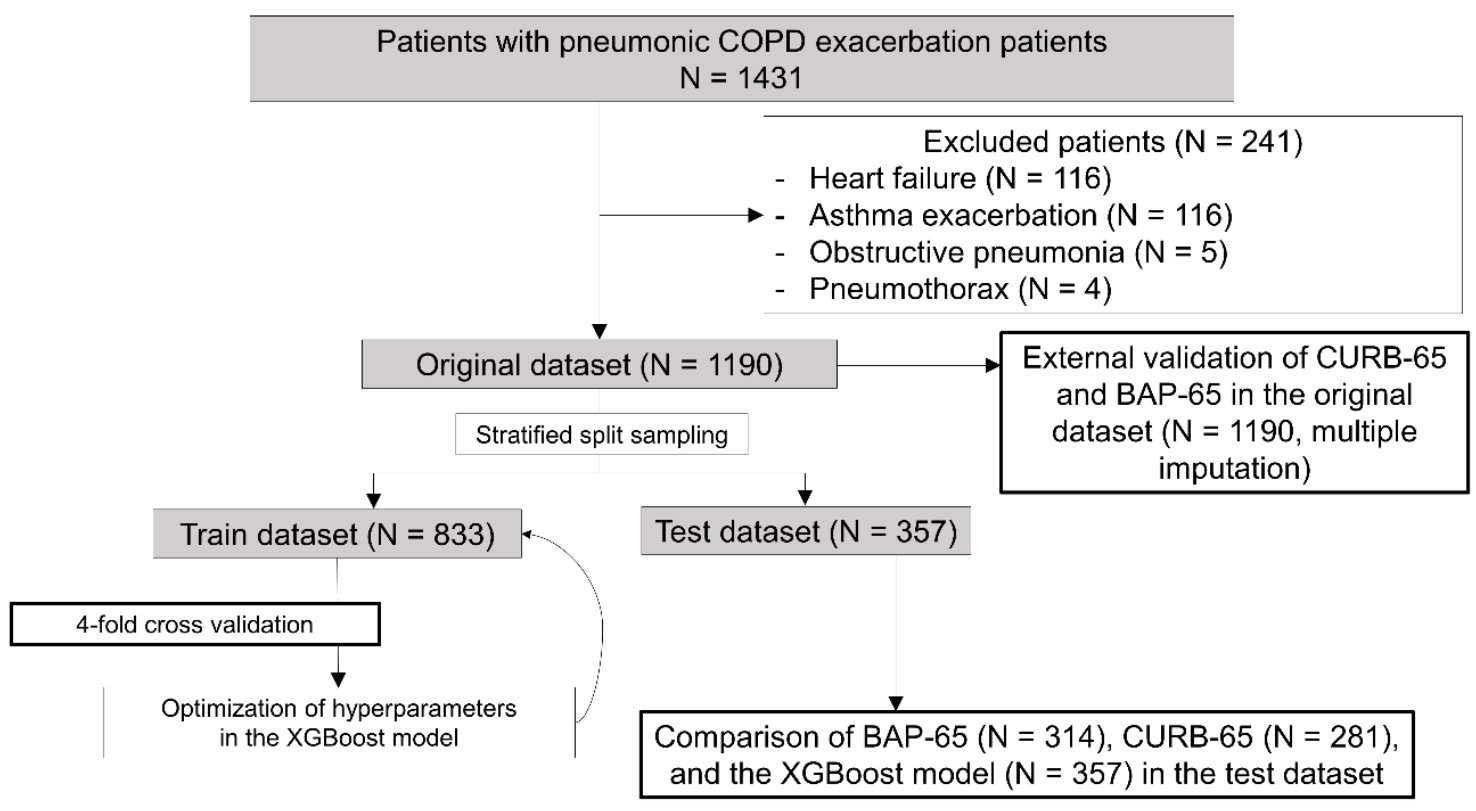

Figure 1. Patient selection flow and framework of the study process

COPD, chronic obstructive pulmonary disease; XGBoost, eXtreme Gradient Boosting 


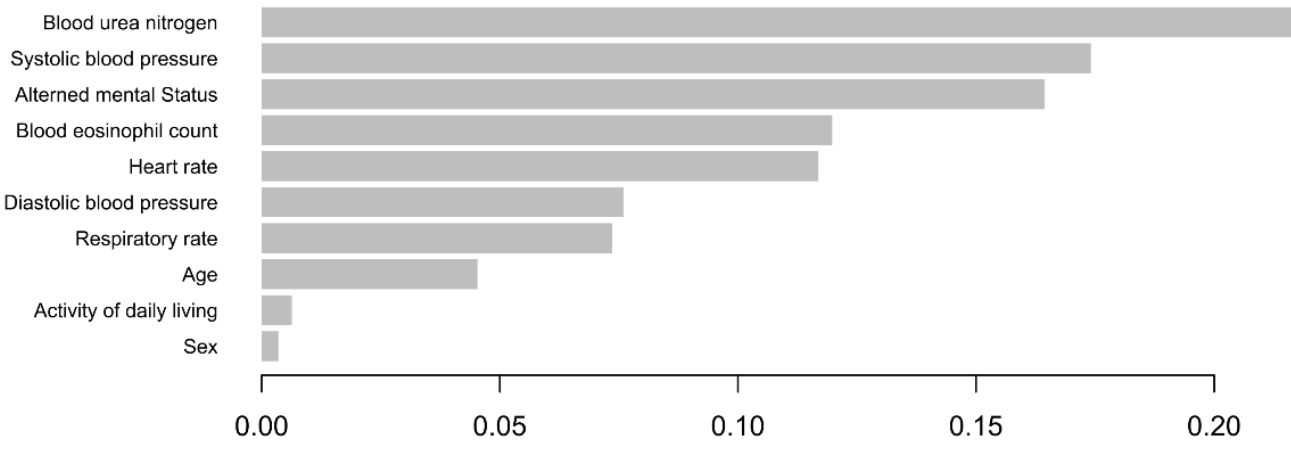

Figure 2. Important variables based on the impurity metric

Blood urea nitrogen was the most important feature. Activities of daily living and sex were of little importance. 


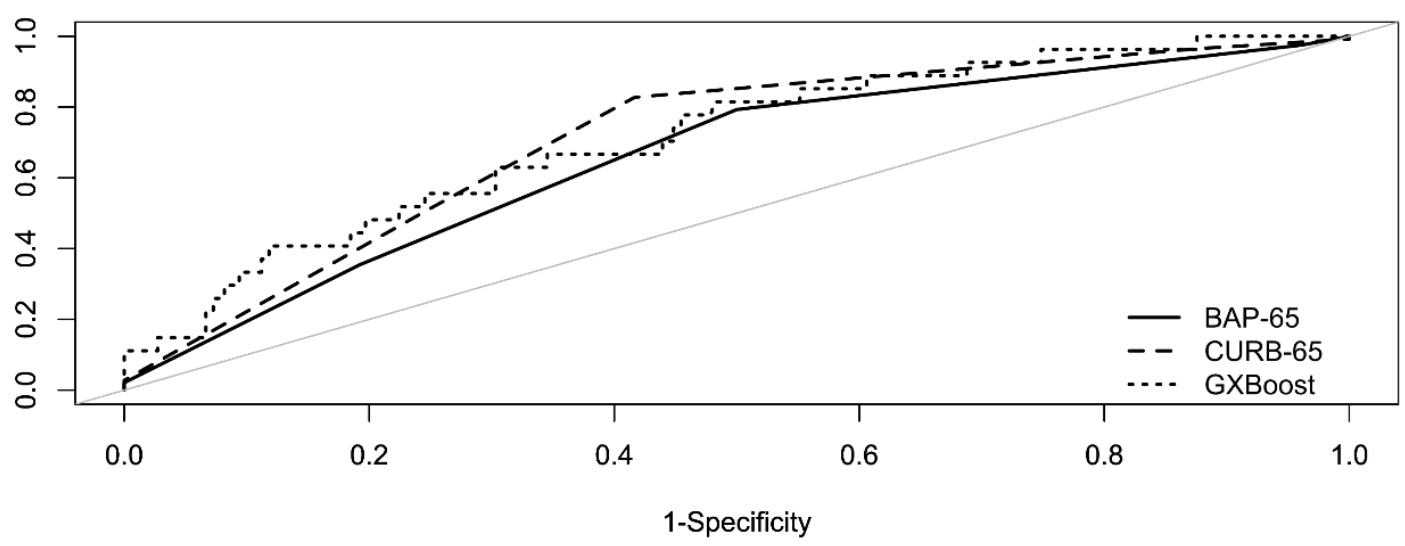

Figure 3. The receiver operating characteristic curves of BAP-65, CURB-65, and the eXtreme Gradient Boosting (XGBoost) model in the test dataset

The XGBoost model showed the best discriminatory performance. 
eTable1. The Transparent Reporting of a multivariable prediction model for Individual Prognosis Or Diagnosis (TRIPOD) checklist

\begin{tabular}{|c|c|c|c|c|}
\hline \multicolumn{3}{|l|}{ Section/Topic } & Checklist Item & Page \\
\hline \multicolumn{5}{|l|}{ Title and abstract } \\
\hline Title & 1 & $\mathrm{D} ; \mathrm{V}$ & $\begin{array}{l}\text { Identify the study as developing and/or validating a multivariable prediction model, the target population, } \\
\text { and the outcome to be predicted. }\end{array}$ & 1 \\
\hline Abstract & 2 & $\mathrm{D} ; \mathrm{V}$ & $\begin{array}{l}\text { Provide a summary of objectives, study design, setting, participants, sample size, predictors, outcome, } \\
\text { statistical analysis, results, and conclusions. }\end{array}$ & 3,4 \\
\hline \multicolumn{5}{|l|}{ Introduction } \\
\hline \multirow{2}{*}{$\begin{array}{l}\text { Background and } \\
\text { objectives }\end{array}$} & $3 \mathrm{a}$ & $\mathrm{D} ; \mathrm{V}$ & $\begin{array}{l}\text { Explain the medical context (including whether diagnostic or prognostic) and rationale for developing } \\
\text { or validating the multivariable prediction model, including references to existing models. }\end{array}$ & 5 \\
\hline & $\beta b$ & $\mathrm{D} ; \mathrm{V}$ & $\begin{array}{l}\text { Specify the objectives, including whether the study describes the development or validation of the } \\
\text { model or both. }\end{array}$ & 5 \\
\hline \multicolumn{5}{|l|}{ Methods } \\
\hline \multirow{2}{*}{ Source of data } & $4 \mathrm{a}$ & $\mathrm{D} ; \mathrm{V}$ & $\begin{array}{l}\text { Describe the study design or source of data (e.g., randomized trial, cohort, or registry data), separately } \\
\text { for the development and validation data sets, if applicable. }\end{array}$ & \\
\hline & $4 \mathrm{~b}$ & $\mathrm{D} ; \mathrm{V}$ & $\begin{array}{l}\text { Specify the key study dates, including start of accrual; end of accrual; and, if applicable, end of follow- } \\
\text { up. }\end{array}$ & 6 \\
\hline \multirow[t]{2}{*}{ Participants } & $5 \mathrm{a}$ & $\mathrm{D} ; \mathrm{V}$ & $\begin{array}{l}\text { Specify key elements of the study setting (e.g., primary care, secondary care, general population) } \\
\text { including number and location of centres. }\end{array}$ & 6 \\
\hline & $5 \mathrm{~b}$ & $\mathrm{D} ; \mathrm{V}$ & Describe eligibility criteria for participants. & 6 \\
\hline
\end{tabular}




\begin{tabular}{|c|c|c|c|c|}
\hline & $5 \mathrm{c}$ & $\mathrm{D} ; \mathrm{V}$ & Give details of treatments received, if relevant. & Not applicable \\
\hline \multirow{2}{*}{ Outcome } & $6 a$ & $\mathrm{D} ; \mathrm{V}$ & Clearly define the outcome that is predicted by the prediction model, including how and when assessed. & 6 \\
\hline & $\mathrm{sb}$ & $\mathrm{D} ; \mathrm{V}$ & Report any actions to blind assessment of the outcome to be predicted. & 6 \\
\hline \multirow[t]{2}{*}{ Predictors } & $7 \mathrm{a}$ & $\mathrm{D} ; \mathrm{V}$ & $\begin{array}{l}\text { Clearly define all predictors used in developing or validating the multivariable prediction model, } \\
\text { including how and when they were measured. }\end{array}$ & 6 \\
\hline & $7 \mathrm{~b}$ & $\mathrm{D} ; \mathrm{V}$ & Report any actions to blind assessment of predictors for the outcome and other predictors. & 6 \\
\hline Sample size & 8 & $\mathrm{D} ; \mathrm{V}$ & Explain how the study size was arrived at. & 6 \\
\hline Missing data & 9 & $\mathrm{D} ; \mathrm{V}$ & $\begin{array}{l}\text { Describe how missing data were handled (e.g., complete-case analysis, single imputation, multiple } \\
\text { imputation) with details of any imputation method. }\end{array}$ & 7,8 \\
\hline \multirow{5}{*}{$\begin{array}{l}\text { Statistical } \\
\text { analysis methods }\end{array}$} & 0a & $\mathrm{D}$ & Describe how predictors were handled in the analyses. & 7 \\
\hline & $0 \mathrm{~b}$ & $\mathrm{D}$ & $\begin{array}{l}\text { Specify type of model, all model-building procedures (including any predictor selection), and method } \\
\text { for internal validation. }\end{array}$ & 7,8 \\
\hline & $0 \mathrm{c}$ & $\mathrm{V}$ & For validation, describe how the predictions were calculated. & 7 \\
\hline & Od & $\mathrm{D} ; \mathrm{V}$ & Specify all measures used to assess model performance and, if relevant, to compare multiple models. & 7,8 \\
\hline & 0e & $\mathrm{V}$ & Describe any model updating (e.g., recalibration) arising from the validation, if done. & 7,8 \\
\hline Risk groups & 11 & $\mathrm{D} ; \mathrm{V}$ & Provide details on how risk groups were created, if done. & 7,8 \\
\hline $\begin{array}{l}\text { Development vs. } \\
\text { validation }\end{array}$ & 12 & $\mathrm{~V}$ & $\begin{array}{l}\text { For validation, identify any differences from the development data in setting, eligibility criteria, outcome, } \\
\text { and predictors. }\end{array}$ & 6 \\
\hline \multicolumn{5}{|l|}{ Results } \\
\hline \multirow{2}{*}{ Participants } & $3 a$ & $\mathrm{D} ; \mathrm{V}$ & $\begin{array}{l}\text { Describe the flow of participants through the study, including the number of participants with and } \\
\text { without the outcome and, if applicable, a summary of the follow-up time. A diagram may be helpful. }\end{array}$ & 9 \\
\hline & $3 \mathrm{~b}$ & $\mathrm{D} ; \mathrm{V}$ & $\begin{array}{l}\text { Describe the characteristics of the participants (basic demographics, clinical features, available } \\
\text { predictors), including the number of participants with missing data for predictors and outcome. }\end{array}$ & 9 , Table 1 \\
\hline
\end{tabular}




\begin{tabular}{|c|c|c|c|c|}
\hline & $3 \mathrm{c}$ & $\mathrm{V}$ & $\begin{array}{l}\text { For validation, show a comparison with the development data of the distribution of important variables } \\
\text { (demographics, predictors and outcome). }\end{array}$ & 9 , Table 1 \\
\hline \multirow{2}{*}{$\begin{array}{l}\text { Model } \\
\text { development }\end{array}$} & $4 \mathrm{a}$ & $\mathrm{D}$ & Specify the number of participants and outcome events in each analysis. & 8 \\
\hline & $4 \mathrm{~b}$ & $\mathrm{D}$ & If done, report the unadjusted association between each candidate predictor and outcome. & Not applicable \\
\hline \multirow{2}{*}{$\begin{array}{l}\text { Model } \\
\text { specification }\end{array}$} & $5 \mathrm{a}$ & $\mathrm{D}$ & $\begin{array}{l}\text { Present the full prediction model to allow predictions for individuals (i.e., all regression coefficients, } \\
\text { and model intercept or baseline survival at a given time point). }\end{array}$ & 9 \\
\hline & $5 b$ & $\mathrm{D}$ & Explain how to the use the prediction model. & 9 \\
\hline $\begin{array}{l}\text { Model } \\
\text { performance }\end{array}$ & 16 & $\mathrm{D} ; \mathrm{V}$ & Report performance measures (with CIs) for the prediction model. & 9,10 \\
\hline Model-updating & 17 & $\mathrm{~V}$ & If done, report the results from any model updating (i.e., model specification, model performance). & 9 \\
\hline \multicolumn{5}{|l|}{ Discussion } \\
\hline Limitations & 18 & $\mathrm{D} ; \mathrm{V}$ & $\begin{array}{l}\text { Discuss any limitations of the study (such as nonrepresentative sample, few events per predictor, missing } \\
\text { data). }\end{array}$ & 11,12 \\
\hline \multirow{2}{*}{ Interpretation } & $9 \mathrm{a}$ & $\mathrm{V}$ & $\begin{array}{l}\text { For validation, discuss the results with reference to performance in the development data, and any other } \\
\text { validation data. }\end{array}$ & 10 \\
\hline & $9 \mathrm{~b}$ & $\mathrm{D} ; \mathrm{V}$ & $\begin{array}{l}\text { Give an overall interpretation of the results, considering objectives, limitations, results from similar } \\
\text { studies, and other relevant evidence. }\end{array}$ & 10 \\
\hline Implications & 20 & $\mathrm{D} ; \mathrm{V}$ & Discuss the potential clinical use of the model and implications for future research. & 11,12 \\
\hline \multicolumn{5}{|l|}{ Other information } \\
\hline $\begin{array}{l}\text { Supplementary } \\
\text { information }\end{array}$ & 21 & $\mathrm{D} ; \mathrm{V}$ & $\begin{array}{l}\text { Provide information about the availability of supplementary resources, such as study protocol, Web } \\
\text { calculator, and data sets. }\end{array}$ & 13 \\
\hline Funding & 22 & $\mathrm{D} ; \mathrm{V}$ & Give the source of funding and the role of the funders for the present study. & 2 \\
\hline
\end{tabular}


*Items relevant only to the development of a prediction model are denoted by $\mathrm{D}$, items relating solely to a validation of a prediction model are denoted by $\mathrm{V}$, and items relating to both are denoted $\mathrm{D} ; \mathrm{V}$. 
eFigure1: The validated patient selection algorithm based on the $10^{\text {th }}$ revision of the International Statistical Classification of Diseases and Related Health Problems (ICD-10)

- The admission precipitating the diagnosis of pneumonia (J12, J13, J14, J15, J16, J69 and P23)

- Comorbidities present at the time of admission for COPD (J44.1 and J44.9)
- The admission precipitating the diagnosis of COPD exacerbation (J44.1)

- Comorbidities present at the time of admission for pneumonia (J12, J13, J14, J15, J18, J69, and $\mathrm{P} 23$ )

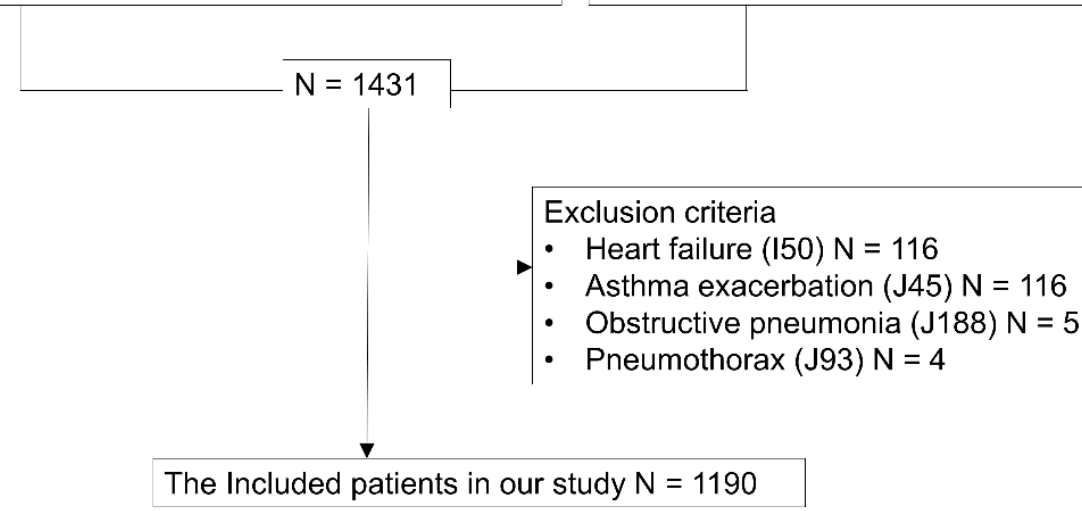


eFigure2: The detailed description of the eXtreme Gradient Boosting algorithm

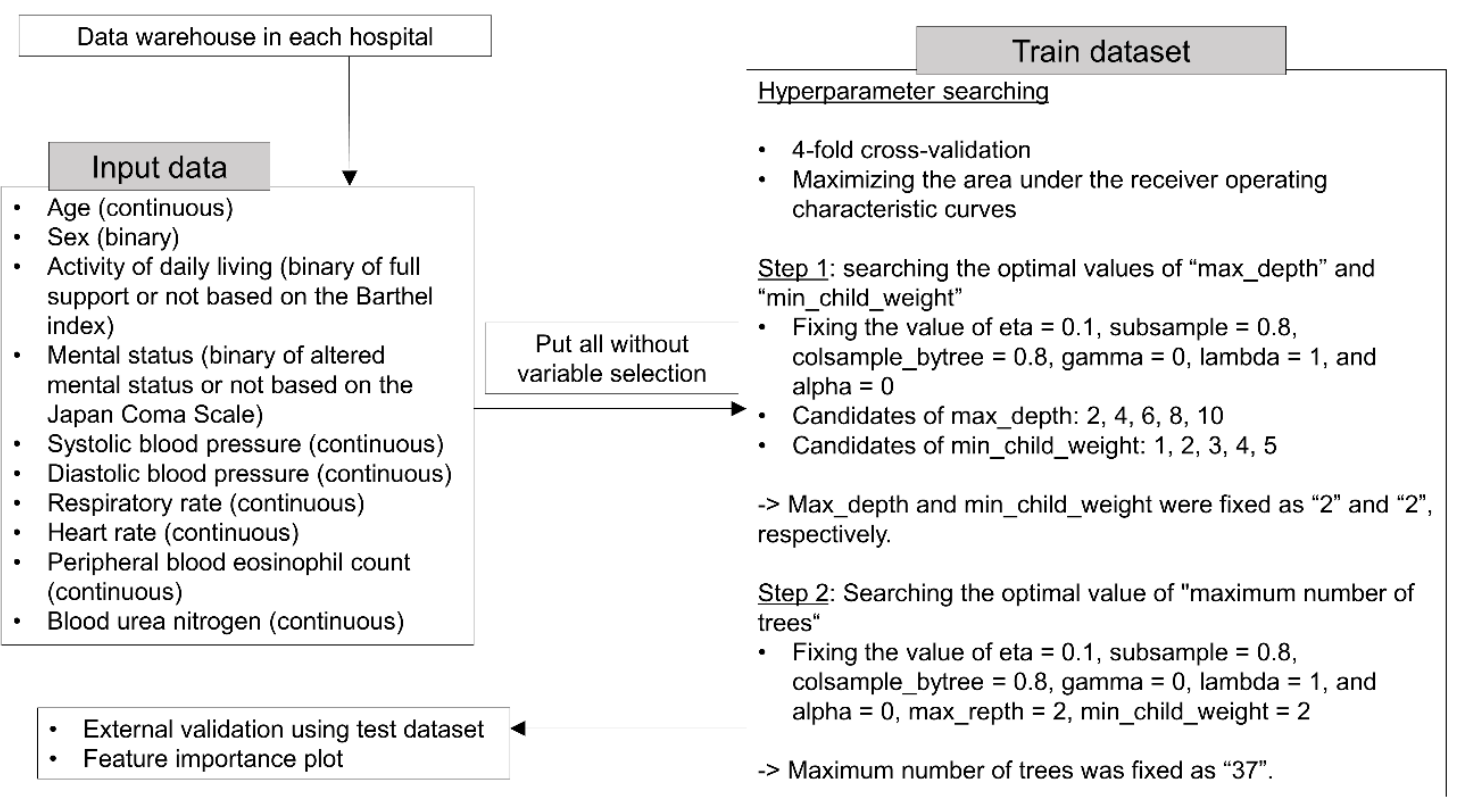

